\title{
ATENDIMENTO AO ESTUDANTE - recepção, SAPP, NAI e UFGInclui
}

\author{
Sandra Aparecida Benite-Ribeiro ${ }^{1}$ \\ Sandra Nara da Silva Novais ${ }^{2}$ \\ Aurélia Magalhães de Oliveira Souza ${ }^{3}$ \\ Joana D'arc Moreira ${ }^{4}$ \\ Vanderlei Balbino da Costa ${ }^{5}$
}

\begin{abstract}
RESUMO: Com o objetivo de analisar o acolhimento e o apoio ao estudante na UFG - Regional Jataí (REJ), no presente estudo avaliamos quais os projetos ou programas de apoio realizados durante o ingresso do estudante e outros serviços de apoio estudantil. Os dados foram coletados de arquivos da Coordenação de Graduação, do Serviço de apoio psicológico e psicopedagógico (SAPP), do Núcleo de acessibilidade e inclusão (NAI), de órgãos Centro de Seleção e do Sistema Integrado de Núcleos de Acessibilidade (SINACE). Em relação à recepção ao ingressante não encontramos um programa institucionalizado, apenas ações isoladas, o que demonstra a importância de institucionalizar essa ação. $\mathrm{O}$ SAPP demonstrou ser eficiente em relação ao atendimento dos estudando, mas deficiente em relação ao número de profissionais e à estrutura física de trabalho destinado ao atendimento da comunidade pelo SAPP. Pela avaliação do NAI desde sua criação, observamos que as ações dedicadas à acessibilidade e inclusão vêm crescendo e obtendo importantes aquisições, tanto materiais quanto de recursos humanos, de forma que nenhum estudante da Regional, que solicitou atendimento pelo NAI, não está sem o devido apoio. Por outro lado, o programa do UFGInclui ainda está iniciando e precisa de auxílio significativo dos órgãos superiores da Universidade para seu efetivo atendimento aos estudantes. Apesar disso, todos os estudantes desses grupos possuem bolsas de permanência e com a ajuda dos próprios estudantes quilombolas e indígenas, a coordenação do programa tem conseguido atender pelo menos as necessidades básicas de todos indígenas e quilombolas da UFG - Regional Jataí.
\end{abstract}

Palavras-chave: Acessibilidade. Inclusão. Gestão escolar.

\section{STUDENT'S SUPPORT SERVICE - Welcoming, SAPP, NAI and UFGInclui}

ABSTRACT: In the present study we evaluated support and assistance programs conducted during the incoming of freshmen and other students' support services programs at the Universidade Federal de Goiás, Regional Jataí (UFG/REJ). The analyzed data were made available by the Coordination of Undergraduate Studies, the Psychological and Psychopedagogical Support Service (SAPP), the Accessibility and

\footnotetext{
${ }^{1}$ Pós-doutorado pela Universidade do Porto, FADEUP, Portugal, em Fisiologia. Doutorado e mestrado em Ciências Biológicas (Zoologia) pela Universidade Estadual Paulista Júlio de Mesquita Filho. Graduado em Ciências Habilitação em Biologia pela Universidade Estadual Paulista Júlio de Mesquita Filho. Professora da Regional Jataí UFG sandrabenite@gmail.com

${ }^{2}$ Doutorado em Educação pela Universidade Federal de São Carlos. Mestrado em História Região e Identidades pela Universidade Federal de Mato Grosso do Sul / Campus de Dourados. Graduação em História Licenciatura Plena pela Universidade Federal de Mato Grosso do Sul / Campus de Aquidauana. Professora da Regional Jataí - UFG novaesnara@ hotmail.com

${ }_{3}^{3}$ Psicóloga e psicopedagoga do Serviço de Apoio Psicológico e Psicopedagógico da UFG. aureliapsi@ @otmail.com

${ }^{4}$ Psicóloga e psicopedagoga do Serviço de Apoio Psicológico e Psicopedagógico da UFG. joanapsico2015@gmail.com

${ }^{5}$ Doutor em Educação Especial - UFSCar/SP, mestre em Educação e graduação em História (UFMT). Professor da Regional Jataí - UFGprofvanderleiufg@gmail.com
} 
Inclusion Center (NAI), the Selection Center and the Integrated System of Accessibility Centers (SINACE). Concerning freshmen's reception, we did not find any institutionalized program, but isolated actions, which demonstrate the importance to institutionalize these types of programs. SAPP was efficient in relation to students' assistance, but deficient concerning the number of professionals and workplace physical structure destined to their program. Since NAI's creation, we have observed that actions dedicated to both accessibility and inclusion have been thriving, in both material and human resource matters, so that every UFG/REJ student, who requested its assistance, has received proper support. On the other hand, NAI program is still crawling and needs significant attention from UFG/REJ's direction board to achieve students' effective assistance and support. In spite of this, all students from these groups have permanence scholarships and the program coordination has managed to assist UFG/REJ quilombola and indigenous students' basic needs.

Key-words: Accessibility. Inclusion. School Management

\section{INTRODUÇÃ̃o}

De acordo com o Instrumento de Avaliação Institucional Externa do INEP-MEC de 2014:

No Eixo "Políticas Acadêmicas" analisam-se os elementos constitutivos das práticas de ensino, pesquisa e extensão, considerando como meta o aprendizado. Enfatiza-se também a relação entre as políticas acadêmicas, a comunicação com a sociedade e o atendimento ao discente. Este Eixo contempla as dimensões 2 (Políticas para o Ensino, a Pesquisa e a Extensão), 4 (Comunicação com a Sociedade) e 9 (Políticas de Atendimento aos Discentes) do Sinaes...." (MinistÉrIO DA EDUCAÇÃO, 2014)

Na Regional Jataí da Universidade Federal de Goiás há programas e projetos de atendimento ao estudante coordenados pela Coordenação de Assuntos da Comunidade Estudantil (CACOM) e pela coordenação de Graduação (COGRAD). Os programas da CACOM englobam as bolsas: Moradia, Permanência, Concessão de Passagens para Eventos Acadêmicos, Acompanhamento de Transporte Municipal e o Restaurante Universitário/Bolsa Alimentação (UNIVERSIDADE FEDERAL DE GOIÁs, 2018). A COGRAD coordena os programas de Recepção ao Ingressante, o Serviço de Apoio Psicológico e Psicopedagógico, o Núcleo de Acessibilidade e Inclusão e o programa UFGInclui. A seguir, serão apresentados os programas coordenados pela COGRAD, seus objetivos, metas, recursos humanos e resultados obtidos até o ano de 2016 por cada um dos programas. 


\section{OBJETIVOS}

O objetivo do presente estudo foi analisar os programas de acolhimento e de apoio ao estudante realizados pela Coordenação de Graduação da Regional Jataí (REJ).

\section{MÉTODOS}

Para o levantamento dos dados foram analisados os documentos presentes da Coordenação de Graduação, no Serviço de Apoio Psicológico e Psicopedagógico e no Núcleo de Acessibilidade e Inclusão nos anos de 2008 a 2016. Foram também aplicados os questionários aos coordenadores de curso, nos quais era perguntado ao coordenador sobre qual o curso e quais as atividades realizadas a cada ano para a recepção dos ingressantes. Os dados compilados no estudo do NAI foram obtidos nos órgãos Centro de Seleção; Sistema Integrado de Núcleos de Acessibilidade (SINACE); NAI/REJ (Dados referentes aos alunos com deficiência).

Os resultados e discussão de cada estudo são apresentados separadamente, como estudo 1, 2, 3 e 4 .

\section{RESULTADOS E DISCUSSÃO}

\section{Estudo 1: Recepção ao Ingressante}

A partir da análise documental, observamos que a Coordenação de Graduação realizou eventos esporádicos durante o período analisado, portanto não institucionalizado. Em 2016 houve uma recepção, em um evento que contou com a colaboração da Coordenação de Extensão e Cultura e com a Direção da REJ. Houve palestras informativas proferidas pelos: Reitor da UFG, Diretor da REJ, Coordenadora de Assuntos Estudantis, Coordenadora de Graduação, uma médica infectologista do curso de Medicina. Os temas das palestras foram relacionados à estrutura e funcionamento da UFG, bolsas de auxílio aos estudantes e doenças sexualmente transmissíveis. Houve também atividades culturais de dança, atividade física e musical. Foi realizada uma 
recepção específica para os alunos indígenas e quilombolas com o coordenador do UFGInclui da UFG, a coordenadora de Graduação da Regional, o coordenador do Núcleo de Acessibilidade e Inclusão e a coordenadora do UFGInclui da UFG - Regional Jataí.

Entre os coordenadores de curso foi averiguado que 9 entre os 25 cursos da Regional desenvolviam alguma atividade que pode ser considerada como um programa de acolhimento ao estudante. As atividades variavam entre palestras, "semana da calourada", aula inaugural, aula magna etc. Quatro coordenadores de curso declararam fornecer orientações ao ingressante somente em disciplinas do primeiro semestre letivo do estudante, de forma que não foram considerados como programa de acolhimento. Dessa forma, consideramos que o programa de acolhimento ao estudante era insipiente e não institucionalizado até o ano de 2016 na REJ.

\section{Estudo 2 SAPP (Serviço de Apoio Psicológico e Psicopedagógico)}

O apoio psicológico e psicopedagógico foi implantado na REJ somente no ano de 2010, com a chegada de técnicos com formação em psicologia e psicopedagogia. Neste momento foi criado um Núcleo, que tinha como objetivo auxiliar o desenvolvimento do processo educativo e que centralizava as funções de apoio psicológico e pedagógico à comunidade interna do CAJ. A partir de então foi criado o Núcleo de Apoio Psicopedagógico (NAPP) que foi implantado após ser aprovado pelo Conselho Diretor, realizado dia 06 de abril de 2011.

O NAPP tinha como objetivo geral apoiar a Direção e as Assessorias de Graduação e Pós-Graduação no desenvolvimento do processo educativo do CAJ/UFG. Os objetivos específicos eram:

- Levantar as fragilidades, no que diz respeito aos aspectos pedagógicos e psicológicos, que interferem no processo de ensino-aprendizagem;

- $\quad$ Estudar medidas que visem contribuir para o desenvolvimento do processo educativo;

- $\quad$ Elaborar projetos de ação para apoio pedagógico e psicológico; 
- $\quad$ Promover cursos e palestras sobre temas afins ao núcleo;

- Desenvolver e coordenar projetos de pesquisa e extensão pertinentes ao NAPP;

- $\quad$ Receber demandas da direção, das coordenações, dos docentes e dos discentes no que diz respeito aos aspectos pedagógicos e psicológicos, que comprometam o processo de ensino-aprendizagem.

Neste projeto também estavam presentes as atribuições de cada um dos profissionais daquela época. Sendo as seguintes:

O Pedagogo devia acompanhar as atividades de ensino, pesquisa, extensão; assessorando-as quanto aos aspectos pedagógicos; sugerindo melhoras para os processos educativos, desenvolvendo projetos de pesquisa e extensão, a fim de apoiar a educação integral dos acadêmicos. Orientava coletiva ou individualmente os docentes, discentes e/ou técnicoadministrativos, mediante situações percebidas por estes, de dificuldades no processo de ensinoaprendizagem.

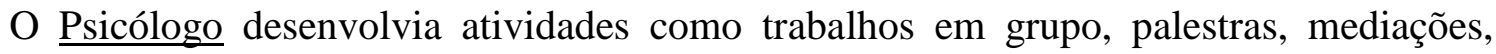
junto às coordenações, docentes, discentes e técnico - administrativos e encaminhava, quando pertinente, para atendimentos e orientações individuais ou em grupos.

Desde sua implantação, o NAPP passou por algumas mudanças, principalmente, no que diz respeito ao modo de as profissionais intervirem junto aos cursos e assessorias e na melhor maneira de ocorrer os encaminhamentos.

Em abril de 2013, a pedagoga pediu exoneração do cargo. A partir daí a psicóloga continuou sozinha sua atuação no NAPP e realizou uma parceria com uma docente do curso de Pedagogia para que atendesse às demandas urgentes na área pedagógica dos discentes. Houve a necessidade de fazer parceria também com o curso de Psicologia, pois o número de atendimentos psicológicos aumentou em demasia.

No ano de 2015 houve novo concurso para preenchimento da vaga, no qual solicitava-se formação em Psicopedagogia e/ou Orientação Educacional para o candidato. Ainda em 2015, devido a mudança no Regimento da REJ, o NAPP (Núcleo de Apoio Psicopedagógico) passou a ser SAPP - Serviço de Apoio Psicológico e Psicopedagógico. 
Atualmente, a psicóloga realiza atendimentos individuais breves, participa de palestras e mesas redondas sobre temáticas diversas que compõem o ambiente acadêmico, faz intervenções in loco com os servidores da REJ, é membro da equipe multidisciplinar e da equipe de capacitação da instituição, oferece orientações para docentes, discentes, técnico-administrativos e terceirizados no que diz respeito à sua área de atuação, dentre outras questões que envolvam a temática saúde do trabalhador.

Sobre a psicologia clínica Macedo (1984) esclarece:

\begin{abstract}
"Entendemos que a psicologia clínica se distingue das demais áreas psicológicas muito mais por uma maneira de pensar e atuar, do que pelos problemas que trata. O comportamento, a personalidade, as normas de ação e seus desvios, as relações interpessoais, os processos grupais, evolutivos e de aprendizagem, são objeto de estudo não só de muitos campos da psicologia como também das ciências humanas em geral" (Macedo, 1994, p.8).
\end{abstract}

Em $1^{\circ}$ de dezembro de 2015, o SAPP recebeu uma profissional para o cargo de pedagogia, área de atuação em psicopedagogia. Esta técnica tem como designação: a) realizar intervenção psicopedagógica junto aos discentes, de forma individual e/ou grupal, quanto às diferentes dificuldades acadêmicas e /ou de aprendizagem, relacionadas à sua modalidade de aprender, considerando os seguintes aspectos: baixa autoestima, ansiedade, tipo de personalidade, déficit de atenção, hiperatividade, memória, fala, leitura, escrita, interpretação, raciocínio, dúvidas quanto ao curso escolhido, adaptação, desânimo acentuado ao estudar, entre outras; b) orientar docentes colaborando no que se refere às metodologias e formas avaliativas aplicadas aos discentes, priorizando os aspectos emocionais e cognitivos da aprendizagem; c) executar ações e projetos educacionais, primando pela saúde emocional do discente e pela melhoria do processo ensino-aprendizagem; d) elaborar projetos de capacitação profissional referente à sua área de atuação, quando necessário; e) participar de projetos elaborados por outros setores da instituição, quando estes necessitarem de orientação psicopedagógica.

De acordo com PORTO (2006 p.107) “A psicopedagogia é uma área de estudo nova, voltada para o atendimento de sujeitos que apresentam problemas de aprendizagem.” (...) Este autor segue afirmando que "cabe à Psicopedagogia o objetivo de resgatar uma visão mais 
globalizante do processo de aprendizagem e dos problemas desses processos". Dessa forma, é indispensável conhecer e pensar sobre os recursos que a psicopedagogia emprega para detectar problemas de aprendizagem e as respectivas intervenções na universidade.

Para solicitar o atendimento ao SAPP, discente, docente e técnicos administrativos enviam e-mail, ligam ou procuram pessoalmente. Não há necessidade de os discentes serem encaminhados pelos professores, mas muitos têm chegado ao Apoio dessa forma. Os docentes têm buscado com mais frequência os atendimentos, seja na área psicológica, seja psicopedagógica.

Devido ao grande número de discentes que buscam apoio psicológico e psicopedagógico, nem sempre é possível realizar tratamento no SAPP. As servidoras fazem os primeiros atendimentos - orientações e, quando necessário, encaminham os discentes para outros profissionais da comunidade externa ou interna (Programa Saudavelmente - UFG/Goiânia).

Alguns casos em que o discente necessita de um acompanhamento mais prolongado e não tem condições financeiras para custear a despesas, nem possui plano de saúde, as profissionais do SAPP encaminham-nos para o Serviço de Psicologia Aplicada (SPA), onde são acompanhados por alunos estagiários do curso de psicologia que são supervisionados por seus professores.

\section{Estudo 3: NAI - Núcleo de Acessibilidade e Inclusão}

Com a promulgação da política nacional de educação na perspectiva da educação especial inclusiva, (BRASIL 2008), foi recomendado que todas as instituições Federais de ensino superior criassem núcleos de acessibilidade. Na UFG - Regional Jataí, o núcleo foi reativado em janeiro de 2014. Partindo dessa premissa, cumpre-nos de forma sucinta conceituar o que é o NAI: O NAI é um canal de comunicação entre a comunidade acadêmica, técnicos administrativos, professores e a direção da UFG -Regional Jataí.

Entre as tantas funções que o núcleo se ocupa em desenvolver, destaca-se o apoio de forma especializada todos os profissionais com deficiência que atuam nas 02 unidades, Riachuelo e Jatobá, constituindo equipe qualificada. Cabe também ao NAI a captação de recursos humanos 
habilitados nas diferentes necessidades educativas especiais, visando apoiar a inclusão de pessoas com deficiência na comunidade acadêmica (estudantes, técnico-administrativos e docentes). Ao reativar o núcleo de acessibilidade nesta regional, tivemos a oportunidade de:

- Propor políticas educacionais inclusivas que possibilitem o acesso e permanência dos estudantes no ensino superior;

- Promover e acompanhar o desenvolvimento acadêmico dos estudantes com deficiência;

- Apoiar a inclusão dos estudantes, técnico-administrativos e professores com deficiência nas atividades de ensino, pesquisa e extensão;

- Identificar as barreiras que restringem a autonomia, a liberdade e a individualidade das pessoas com deficiência ou mobilidade reduzida.

Ao longo de aproximadamente 04 quatro anos o NAI em parceria com a direção, SINACE, e Pró-Reitorias alcançou algumas metas que favoreceram a inclusão e a permanência dos estudantes com deficiência nesta regional. Nesse sentido, pouco a pouco, a coordenação do NAI tem proposto e alcançando adequações visando a superação de barreiras:

- Físicas: rampas; barra de apoio; corrimão; piso com sinalização tátil; sinalizadores; alargamento de portas e vias; instalação de elevadores; dentre outras.

- Pedagógicas: eliminação de barreiras pedagógicas que flexibilizam o currículo, como por exemplo, avaliação diferenciada; capacitação docente; formação continuada; apoio aos candidatos durante o ENEM e aos estudantes de graduação e de pós-graduação).

- Atitudinais: promoção de debates e eventos, como seminários, palestras que possam mudar as atitudes de funcionários, professores e demais seguimentos dessa regional no sentido de mudar seu comportamento atitudinal em relação as pessoas com deficiência. Ou seja, respeito às diferentes necessidades educativas especiais. cultura surda, questões étnico-raciais, dentre outras. 
Até o ano de 2016 há matriculados nesta regional aproximadamente 35 estudantes com deficiência e/ou mobilidade reduzida total ou parcial que direto o indiretamente são atendidos pela equipe que compõem o núcleo de acessibilidade. 
Tabela 1 - Número de Alunos com deficiência na Regional Jataí, UFG

\begin{tabular}{lllll}
\hline Deficiência & Ano & Ano & Ano & $\begin{array}{l}\text { Ano } \\
\text { Física }\end{array}$ \\
& 2014 & 2015 & 2016 & 2017 \\
& 10 & 07 & 09 & 11 \\
& alunos & alunos & alunos & alunos \\
\hline Visual & 03 & 03 & 15 & 15 \\
& alunos & alunos & alunos & alunos \\
\hline Auditiva & 05 & 04 & 06 & 07 \\
& alunos & alunos & alunos & alunos \\
\hline Intelectual & 03 & 03 & 01 & 02 \\
& alunos & alunos & alunos & alunos \\
\hline Total & 21 & 17 & 31 & 35 \\
& alunos & alunos & alunos & alunos \\
\hline
\end{tabular}

Fonte: os autores, 2018

Para atendimento dos estudantes e servidores com deficiências, há vinculado ao núcleo de acessibilidade e inclusão dessa regional diversos profissionais. Até o ano de 2017 a equipe era composta de: 1 coordenador, 6 intérpretes de libras e 5 bolsistas. Com o ingresso de estudantes e servidores com deficiências, há a previsão de mais profissionais e bolsistas no NAI.

Há de se considerar que o processo de inclusão escolar das pessoas com deficiência muito se apoia para além dos recursos humanos e equipamentos específicos. Assim, temos também a necessidade de investir em recursos tecnológicos que visem apoiar a inclusão desses no ensino superior, a saber: o Sara - Leitor de livros, Leitor multimídia, Lupa, computadores de mesa e notebooks.

Para além das atividades já descritas, são executados os seguintes projetos:

\section{- $\quad$ Pesquisa}

Políticas de ação afirmativa na universidade: possibilidade de inclusão no ensino superior (2014-2018) - $128 \mathrm{~h}$ anual;

\section{- Extensão}

Projeto de Acessibilidade e Inclusão (2016/2018) - 128 h anual;

A formação docente na perspectiva da educação especial inclusiva (2017) - 128 h anual; 
IV Seminário de Acessibilidade e Inclusão no ensino superior (2017) - 64 h anual; A educação especial inclusiva na perspectiva da literatura imagética (2017) - 128 h anual;

Ações de acessibilidade.

$1^{\circ}$ Seminário de Acessibilidade e Inclusão da Regional Jataí (2014) - 69 participantes;

$2^{\circ}$ Seminário de Acessibilidade e Inclusão da Regional Jataí (2015) - 102 participantes;

$3^{\circ}$ Seminário de Acessibilidade e Inclusão da Regional Jataí (2016) - 37 participantes;

$4^{\circ}$ Seminário de Acessibilidade e Inclusão da Regional Jataí (2017) - 200 participantes

As principais conquistas obtidas pelo NAI até o ano de 2017 foram:

- $\quad$ Rampas de acesso à universidade;

- Demarcação de vagas para pessoas com deficiência;

- $\quad$ Carro adaptado;

- Sala própria para o atendimento aos alunos com deficiência matriculados na regional;

- Qualificação e certificação dos intérpretes;

- $\quad$ Instalação de elevador na unidade Riachuelo;

- $\quad$ Piso tátil/direcional.

\section{Estudo 4: Programa UFGInclui}

O Programa UFG Inclui, criado na Universidade Federal de Goiás em 2008, tem entre tantas metas garantir a inclusão e permanência dos estudantes oriundos de escolas públicas, indígenas, negros e quilombolas no espaço universitário. Considerando que essa ação é uma questão de cidadania, o núcleo de acessibilidade e inclusão (NAI), é um parceiro fundamental na realização dessas conquistas, bem como dessas ações inclusivas. É de fundamental importância assinalar que o programa UFG Inclui tem crescido a cada ano.

Em 2016, 84 estudantes indígenas e quilombolas ingressaram nas Regionais Goiânia, Goiás, Catalão e Jataí, o maior número desde a sua criação, em 2009. Em 2015, foram 35. Com o 
ingresso dos novos estudantes, a Universidade Federal de Goiás (UFG) conta agora com mais de 60 indígenas e mais de 100 quilombolas.

Cumpre-nos ressaltar que o UFG Inclui prevê uma vaga extra em cada curso de graduação em que houver demanda indígena e quilombola, quando oriundos de escola pública, a partir da pontuação obtida pelo Exame Nacional de Ensino Médio (Enem), bem como 15 vagas para candidatos surdos na graduação Letras Libras. Dessa forma, todos os cursos da REJ devem oferecer uma vaga adicional por curso para indígenas e quilombolas, quando há demanda. $\mathrm{O}$ número de alunos matriculados até o ano de 2016, estratificados pelo curso são apresentados na Tabela 2. Todos os indígenas e quilombolas são cadastrados no MEC e recebem uma Bolsa de Permanência com recursos do $\mathrm{MEC}$, na tabela são apresentadas as demais bolsas que são ofertadas pela CACOM/REJ.

Tabela 2 - Número de Alunos Indígenas e Quilombolas da Regional Jataí, UFG, estratificados pelo curso até o ano de 2016.

\begin{tabular}{lll}
\hline Curso & Número de alunos & Bolsas* \\
\hline Medicina & 3 indígenas 3 & 3 bolsas alimentação \\
& quilombolas & \\
\hline Enfermagem & 1 indígena & 1 bolsa alimentação \\
\hline Direito & 1 quilombola & 1 bolsa alimentação e 1 bolsa \\
& & moradia \\
\hline Fisioterapia & 1 indígena e 1 & 2 bolsas alimentação \\
& quilombola & \\
\hline Pedagogia & 1 indígena & moradia \\
& & 1 bolsa alimentação \\
\hline Agronomia & 1 quilombola & MEC. Fonte os autores, 2018
\end{tabular}

A coordenação de graduação promoveu em 2016 e 2017 uma recepção exclusiva aos estudantes indígenas, quilombolas e com deficiência, ingressantes nos diversos cursos dessa regional. Para tanto, promoveu encontros de integração no sentido de poder ambientá-los a 
universidade. Atividades de recepção e de acolhimento dos estudantes indígenas e quilombolas na UFG devem englobar:

- Acompanhamento com monitores específicos dos diferentes componentes curriculares;

- Rodas de conversa com os estudantes sobre ingresso e permanência na UFG a partir das Ações Afirmativas;

- Agenda de encontros e estratégias de comunicação para que as demandas possam ser propostas, encaminhadas e acompanhadas;

- Consolidação de uma estrutura que garanta o cumprimento do direito à educação dos estudantes indígenas e quilombolas na UFG.

- A aprendizagem colaborativa nas redes de ensino;

- Ensino construído por meio das redes colaborativas - união das instituições educacionais;

- Construção de conhecimentos colaborativos - professores ensino comum planejando juntos.

O núcleo de acessibilidade e inclusão, bem como a COGRAD e a Assessória Estudantil devem entender que o processo de ensino e aprendizagem deverá ocorrer de forma colaborativa envolvendo docentes com as mais variadas formações (VYGOTSKY, 1984). Nesse sentido, o ensino/aprendizagem deve ser diferenciada e inovadora às pessoas com deficiência, indígenas e quilombolas com: currículos adaptados, metodologias diferenciadas, recursos didáticos apropriados, procedimentos metodológicos diferenciados;

Como defensores que somos das ações inclusivas, do ensino interdisciplinar e da partilha do saber entre docentes nas diversas áreas do conhecimento, entendemos que aprendizagem colaborativa e cooperativa têm sido a melhor forma de construir espaços educativos aos estudantes com deficiência, indígenas e quilombolas nas diferentes redes de ensino. Precisamos propagar a adoção do conhecimento construído socialmente e compartilhar o conhecimento (VYGOTSKY, 1984).

Além do exposto, considerando que a aprendizagem é uma ação coletiva que envolve diversos profissionais em suas diferentes áreas do saber, acreditamos que a adoção do ensino 
colaborativo por meio da constituição de equipe multidisciplinar constituída por diferentes profissionais que trabalham juntos, planejam juntos, trocam experiências e que quando há interação e mediação entre esses profissionais, todos os estudantes independentemente de sua origem aprendem com maior qualidade. Desse modo, valorizar, construir e produzir saberes previamente apreendidos pelos estudantes em sua realidade, talvez seja a melhor forma de promover a inclusão daqueles que durante séculos foram excluídos de direitos sociais.

\section{CONSIDERAÇÕES FINAIS}

Concluímos, pelo presente, estudo que o programa de acolhimento ao estudante não está institucionalizado e precisa ser urgentemente implantado. Por outro lado, o SAPP tem atendido de forma regular aos estudantes da UFG - Regional Jataí, porém para o pleno funcionamento do SAPP, diante da crescente demanda das casuísticas, faz-se necessário aumentar o quadro de servidores nas áreas de atuação específica. De mesma forma, para que este setor tenha melhores condições de trabalho e atenda mais pessoas, é necessária também a melhoria da estrutura física. O NAI tem crescido exponencialmente desde sua implantação, pois todos estudantes que solicitaram atendimento especial foram devidamente atendidos, de forma que podemos concluir que a REJ vem tentando cumprir com seu papel social de inclusão. Para o atendimento eficiente de pessoas com deficiência física ou mental, ainda são necessárias adequações da estrutura física e humana, tais como: sinalização das duas unidades, Riachuelo e Jatobá; adequação dos espaços essenciais para o ensino, pesquisa e extensão (laboratórios, bibliotecas, salas de aula); aquisição de materiais, tais como programas de computador, lupas, impressora braile, máquina de escrever braile, folheador de livros etc. e melhoria dos recursos humanos por meio de concurso público de intérpretes, pedagogos, ledores e fonoaudiólogos. O programa UFGInclui apresenta uma situação crítica em relação ao espaço físico e equipamentos, pois até o momento não possui um local físico determinado para o atendimento dos discentes. Além disso, faltam recursos humanos, pois até o período desta pesquisa somente a coordenadora do UFGInclui tem que desempenhar todas as tarefas necessárias para atendimento dos estudantes, tarefas essas que não seriam corretamente cumpridas se não fosse o auxílio que os próprios 
estudantes prestam ao grupo. Por outro lado, todos os estudantes possuem as bolsas do MEC e a maioria deles possuem outros tipos de bolsas fornecidos pela CACOM.

\section{REFERÊNCIAS BIBLIOGRÁFICAS}

MACEDO, L. Ensaios Construtivistas. 2a ed. Casa do Psicólogo. São Paulo, 1994.

MINISTÉRIO DA EDUCAÇÃO. Instrumento de Avaliação Institucional Exterma, 2014.

PORTO, Olívia. Psicopedagogia institucional: teoria, prática e assessoramento psicopedagógico. Wak. São Paulo, 2006.

UNIVERSIDADE FEDERAL DE GOIÁS, R. J. CACOM - o que é? Disponível em: <https://ccom.jatai.ufg.br//n/47928-o-que-e>.

VYGOTSKY, LS. A formação social da mente. 3. ed. São Paulo: Martins Fontes, 1984. 